\title{
More pieces of the platelet activation puzzle slide into place
}

\author{
Lawrence F. Brass
}

Departments of Medicine and Pharmacology, University of Pennsylvania School of Medicine, Room 913 BRB-II, 421 Curie Boulevard, Philadelphia, Pennsylvania 19104, USA. Phone: (215) 573-3540; Fax: (215) 573-2189; E-mail: Brass@mail.med.upenn.edu.

Platelets are normally activated at sites of vascular injury by a combination of collagen exposure, local thrombin generation, and the release of ADP from damaged cells and other activated platelets. Drugs targeting ADP receptors have proved to be clinically useful in settings where platelet activation contributes to arterial thrombosis. However, until the past few years, relatively little was known about the mechanisms by which platelets respond to ADP. Indirect evidence suggested that 1 or more G proteins and $G$ protein-coupled receptors were involved, but none of the receptors known to respond to adenine nucleotides appeared to have the necessary pharmacological profile. In vitro, ADP causes platelets to change shape (undergo cytoskeletal reorganization), secrete their storage granules, expose binding sites for fibrinogen on the $\alpha_{I I b} \beta_{3}$ integrin, and aggregate. These events all require an increase in cytosolic $\mathrm{Ca}^{2+}$ concentration and are facilitated by the ability of ADP to inhibit cAMP formation by adenylyl cyclase $(1,2)$.

It now appears that no single receptor can mediate the full response of platelets to ADP. Based on pharmacological studies with selective agonists and antagonists, human platelets seem to display at least 3 distinct receptors for ADP. Two of these, $\mathrm{P}_{2} \mathrm{Y}_{1}$ and $\mathrm{P}_{2} \mathrm{Y}_{\mathrm{AC}}$ (also referred to as P2cyc and a variety of other names), are, as predicted, $G$ protein-coupled receptors (2-5). The third, $\mathrm{P} 2 \mathrm{X}_{1}$, is a $\mathrm{Ca}^{2+}$ channel (6-8). $\mathrm{P} 2 \mathrm{Y}_{\mathrm{AC}}$ has been defined pharmacologically but has not yet

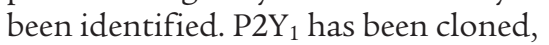
and as reported in the article by Léon and colleagues in this issue of the JCI (9) and in an essentially simultaneous report by Fabre and coworkers (10), has been knocked out in mice.
Studies of platelets from genetically engineered mice are not necessarily informative about human platelets, but in the case of the $2 \mathrm{P}_{2} \mathrm{Y}_{1}$ receptor knockouts, the results are very consistent with those predicted by pharmacological approaches to human platelets. In the absence of $\mathrm{P}_{2} \mathrm{Y}_{1}, \mathrm{ADP}$ is still able to inhibit cAMP formation in mouse platelets, but the ability of ADP to cause an increase in cytosolic $\mathrm{Ca}^{2+}$, shape change, and aggregation is greatly impaired. The mice have a minimal increase in bleeding times and show some resistance to thromboembolic mortality after injection of ADP, but they are not predisposed to spontaneous hemorrhage. Furthermore, despite the presence of $\mathrm{P}_{2} \mathrm{Y}_{1}$ in cells other than platelets, the animals appear to be otherwise normal, and primary responses to platelet agonists other than ADP were unaffected. In combination with serotonin, another weak activator of phospholipase $\mathrm{C}$ in platelets, ADP still promotes aggregation of $\mathrm{P} \mathrm{Y}_{1^{-/-}}$platelets. Thus, the in vitro results with these platelets agree with the data of Kunapuli and coworkers $(4,11)$ who treated human platelets with selective antagonists of $\mathrm{P}_{2} \mathrm{Y}_{1}$.

The identification and deletion of the $\mathrm{P}_{2} \mathrm{Y}_{1}$ receptor helps to define a useful target for antiplatelet drug development. The information also fits within the broader context of efforts to understand the mechanisms by which platelets are activated in vivo. With the notable exception of collagen, most agonists activate platelets by binding to $G$ protein-coupled receptors on the platelet surface. Thus, thrombin binds to and cleaves the $\mathrm{NH}_{2}$ terminus of $2 \mathrm{G}$ protein-coupled receptors, PAR1 and PAR4 (PAR3 and PAR4 on mouse platelets). Similarly, the receptor for the aspirin-sensitive arachidonate metabolite thromboxane
$\mathrm{A}_{2}$ belongs to this class of proteins, as does the $\alpha_{2 \mathrm{~A}}$ adrenergic receptor, which activates platelets in response to epinephrine. Now, the 2 ADP receptors join this list. Collagen, on the other hand, activates platelets via the integrins $\alpha_{2} \beta_{1}$ and $\alpha_{\mathrm{II}} \beta_{3}$, and the cell surface glycoproteins GPIb and GPVI, none of which has an obvious link to $G$ proteins. Some endogenous antiplatelet agents, such as the endothelial cell-derived $\mathrm{PGI}_{2}$, also work via $\mathrm{G}$ protein-coupled receptors. The number of copies of each $G$ protein-coupled receptor on the platelet surface varies, but typically ranges from a few hundred to a few thousand per platelet. The relative abilities of these receptors to cause platelet activation seem to be related less to expression level than to their intrinsic efficiency of coupling to different $G$ proteins.

As illustrated in Figure 1, the various $G$ protein-coupled receptors cause platelet activation by serving as guanine nucleotide exchange factors (GEFs) for heterotrimeric $G$ proteins. Activated receptors allow $G$ proteins to reach the GTP-bound active state by displacing GDP from the G protein's guanine nucleotide-binding site. To a large extent, it is the selection of $G$ proteins that determines which pathways are activated by a particular agonist. Both the GTPbound $\alpha$ subunit of the $G$ protein $\left(G_{\alpha}\right)$ and the heterodimer formed by the $\beta$ and $\gamma$ subunits $\left(G_{\beta \gamma}\right)$ play roles in signal transduction. Human platelets contain at least 10 forms of $G_{\alpha}$, including members of the $G_{i}$ family $\left(G_{i 1 \alpha}, G_{i 2 \alpha}, G_{i 3 \alpha}\right.$, and $\left.G_{z \alpha}\right)$, the $G_{q}$ family $\left(G_{q \alpha}\right.$ and $\left.G_{16 \alpha}\right)$, and the $G_{12}$ family $\left(G_{12 \alpha}\right.$ and $\left.G_{13 \alpha}\right)$, as well as $G_{s \alpha}$. A number of studies, including patient descriptions and some recent knockouts in mice, have helped to define the roles of particular recep- 


\section{Figure1}

Effects of ADP and some of the other relatively well understood thrombotic mediators on signaling in platelets. Most platelet agonists work using G protein-coupled receptors that act as guanine nucleotide exchange factors for 1 or more $G$ proteins. An asterisk next to a receptor or $G$ protein indicates that mice lacking that gene have been created but have not necessarily been studied for platelet function in the absence of the protein. PLC, phospholipase C.

tors, $G$ proteins, and downstream molecules in platelet aggregation. Some of those results are summarized in Figure 1.

A number of principles regarding platelet activation have emerged from those studies. First, agonist potency often seems to correlate best with the ability of the agonist to activate phospholipase C. Strong agonists, such as thrombin, tend to drive phospholipase $\mathrm{C}$ (and, by extension, phosphoinositide hydrolysis) harder than do weaker agonists. Weaker agonists, such as ADP and epinephrine, are more heavily dependent on their simultaneous ability to inhibit cAMP formation. Typically, strong coupling to phospholipase $\mathrm{C}$ is thought to be mediated by the $G$ proteins of the $G_{q}$ family, whereas weaker coupling is activated by the pertussis toxin-sensitive members of the $G_{i}$ family via their $\mathrm{G}_{\beta \gamma}$ heterodimer. However, this general observation oversimplifies matters. In mice lacking $\mathrm{G}_{\mathrm{q} \alpha}$ (apparently the major $\mathrm{G}_{\mathrm{q}}$ family member in mouse platelets), neither thrombin, $\mathrm{ADP}$, or any of the other agonists tested could induce platelet aggregation (12), implying that all of these agonists activate receptors that are coupled to phospholipase $C$ through $\mathrm{G}_{\mathrm{q}}$. Second, agonists that inhibit cAMP formation as well as activating phospholipase $\mathrm{C}$ do so via members of the $\mathrm{G}_{\mathrm{i}}$ family. Of the 4 members of this family expressed in human platelets, $\mathrm{G}_{\mathrm{i} 2 \alpha}$ is the most abundant, and $\mathrm{G}_{\mathrm{z} \alpha}$ is the least similar to the others. Interestingly, recent studies show that these proteins are not functionally interchangeable, because knockouts of $G_{i 2 \alpha}$ and $G_{z \alpha}$ in mice produce distinguishable phenotypes of platelet

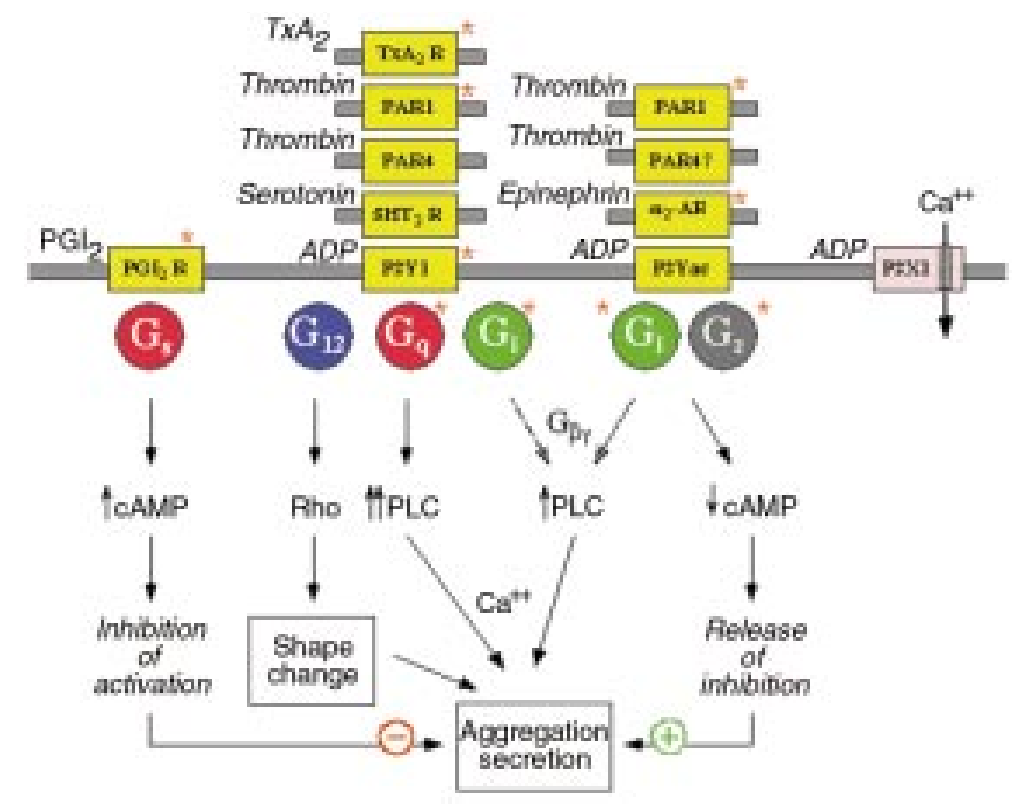

dysfunction. Those studies also suggest additional roles for $G_{i}$ family members beyond the activation of phospholipase $\mathrm{C}$ and inhibition of adenylyl cyclase (J. Yang and L. Brass, unpublished observations). In some cases, such as for thrombin, inactivation of adenylyl cyclase is accomplished using the same receptor that activates phospholipase $\mathrm{C}$, whereas for ADP, the tasks are split between 2 or more receptors. Third, weaker agonists that primarily stimulate phospholipase $\mathrm{C}$ (such as serotonin) can combine with agonists that primarily inhibit adenylyl cyclase (such as epinephrine) to cause platelet activation. In the case of ADP, the effects of $\mathrm{P}_{2} \mathrm{Y}_{1}$ or $\mathrm{P}_{2} \mathrm{Y}_{\mathrm{AC}}$ antagonists can be overcome by combining ADP with an agonist that is coupled only to phospholipase $C$ activation or to adenylyl cyclase inhibition (11). Fourth, for reasons that remain unclear, increasing adenylyl cyclase activity in platelets induces a state of tonic inhibition of platelet activation that can be relieved by reversing the activation of this enzyme. Removal of the inhibition (such as occurs in mice lacking $\mathrm{PGI}_{2}$ receptors) results in increased thrombosis in models of vascular injury (13). Finally, the reorganization of the actin cytoskeleton that is responsible for platelet shape change is probably mediated by a $\mathrm{G}_{12}$ family member. In $\mathrm{G}_{\mathrm{q} \alpha}$ knockout mice, platelet agonists still caused shape change, even though they failed to cause platelet aggregation (12). $G_{12 \alpha}$ null mice have not been reported; lack of $\mathrm{G}_{13 \alpha}$ leads to intrauterine death (14). Recent studies in other cells have shown that p115 Rho-GEF can provide a link between members of the $G_{12}$ family and the members of the Rho family of low molecular weight GTP-binding proteins that regulate actin filament formation. It remains to be seen whether it does so in platelets. Because platelets from $\mathrm{P}_{2} \mathrm{Y}_{1}$ null mice fail to change shape in response to ADP and also fail to aggregate, it is reasonable to think that $\mathrm{P}_{2} \mathrm{Y}_{1}$ is coupled to $G_{12}$ as well as to $G_{q}$.

By their very nature, human platelets are nontransfectable. They are also (inconveniently enough) impenetrable to agents such as pertussis toxin that allow the selective inactivation of some members of the $G_{i}$ subfamily of $G$ proteins. Thus, pharmacological studies with selective receptor agonists and antagonists, combined with selected knockouts of receptors and $G$ proteins (15), have provided many new insights into the earliest events of platelet activation. Many pieces of the puzzle remain. The identity of the ADP receptor that inhibits cAMP formation (and triggers any other events that are mediated by $\mathrm{G}_{i}$ family members) is still unknown. The features 
of the various receptors that are responsible for the differences in efficiency with which they couple to various $G$ proteins and discriminate between members of the same closely related family are still largely unidentified. The mechanism by which cAMP inhibits G protein-coupled signaling, and the basis of the failure of collagen-induced platelet aggregation in mouse platelets lacking $\mathrm{G}_{\mathrm{q}}$ are still poorly understood. Finally, except for phospholipase C, the links from $G$ protein-coupled receptors to the pathways involved in fibrinogen receptor activation, aggregation, and secretion remain mysterious. Our ability to draw large numbers of arrows on maps such as Figure 1 should not obscure the fact that a large number of gaps remains to be filled. In platelet biology, it is often clear where things start and finish, but defining the steps along the way and determining how they are regulated should provide a fruitful subject for research for a number of years to come.

1. Mills, D.C.B. 1996. ADP receptors on platelets. Thromb. Haemost. 76:835-856.

2. Cattaneo, M., and Gachet, C. 1999. ADP receptors and clinical bleeding disorders. Arterioscler Thromb. Vasc. Biol. 19:2281-2285.

3. Léon, C., et al. 1997. The ${\mathrm{P} 2 Y_{1}}_{1}$ receptor is an ADP receptor antagonized by ATP and expressed in platelets and megakaryoblastic cells. FEBS Lett. 403:26-30.

4. Daniel, J.L., et al. 1998. Molecular basis for ADP-induced platelet activation. I. Evidence for three distinct ADP receptors on human platelets. J. Biol. Chem. 273:2024-2029.

5. Jin, J.G., Daniel, J.L., and Kunapuli, S.P. 1998 Molecular basis for ADP-induced platelet activation. II. The P2Y1 receptor mediates ADPinduced intracellular calcium mobilization and shape change in platelets. J. Biol. Chem 273:2030-2034.

6. McKenzie, A.B., Mahout-Smith, M.P., and Sage, S.O. 1996. Activation of receptor-operated channels via P2X1 not P2T purinoreceptors in human platelets. J. Biol. Chem. 271:2879-2881.

7.Vial, C., Hechler, B., Léon, C., Cazenave, J.P., and Gachet, C. 1997. Presence of P2X, purinoceptors in human platelets and megakaryoblastic cell lines. Thromb. Haemost 78:1500-1504.

8. Sun, B., Li, J., Okahara, K., and Kambayashi, J. 1998. P2X1 purinoceptor in human platelets. Molecular cloning and functional characterization after heterologous expression. J. Biol. Chem. 273:11544-11547.

9. Léon, C., et al. 1999. Defective platelet aggregation and increased resistance to thrombosis in purinergic $\mathrm{P}_{2} \mathrm{Y}_{1}$ receptor-null mice. J. Clin. Invest. 104:1731-1737.

10. Fabre, J.-E., et al. 1999. Decreased platelet aggregation, increased bleeding time and resistance to thromboembolism in P2Y1-deficient mice. Nat. Med. 5:1199-1202.

11. Jin, J.G., and Kunapuli, S.P. 1998. Coactivation of two different $G$ protein-coupled receptors is essential for ADP-induced platelet aggregation. Proc. Natl. Acad. Sci. USA. 95:8070-8074.

12. Offermanns, S., Toombs, C.F., Hu, Y.H., and Simon, M.I. 1997. Defective platelet activation in Gq $\alpha$-deficient mice. Nature. 389:183-186.

13. Murata, T., et al. 1997. Altered pain perception and inflammatory response in mice lacking prostacyclin receptor. Nature. 388:678-682.

14. Offermanns, S., Mancino, V., Revel, J.-P., and Simon, M.I. 1997. Vascular system defects and impaired cell chemokinesis as a result of $\mathrm{G} \alpha 13$ deficiency. Science. 275:533-536.

15. Offermanns, S., and Simon, M.I. 1998. Genetic analysis of mammalian G-protein signaling. Oncogene. 17:1375-1381 